\title{
Using synthetic speech for remote access to information
}

\author{
MARY BETH ROSSON \\ Thomas J. Watson Research Center, Yorktown Heights, New York
}

\begin{abstract}
The availability of unlimited text-to-speech synthesis systems provides the potential for remote access to databases over existing telephone systems. This paper describes some of the intelligibility and user interface problems associated with such applications and reports work aimed at understanding and solving these problems. In particular, it describes an approach to intelligibility problems based on the understanding and manipulation of listeners' adaptation to synthetic speech, and user interface work examining use of a simulated travel information system.
\end{abstract}

A promising class of applications for speech synthesis is that of remote access to information. Many electronic databases exist today, but most can be queried only if the user has available a CRT or a teletype terminal. Allowing access to these databases through telephone connections, in which touch-tone keypresses (or in some cases, verbal commands) provide the input, and synthetic speech the output, would expand this information market tremendously. However, although the technology now exists to provide such systems, many behavioral questions remain unanswered.

There are two major classes of behavioral issues. First, we need to address problems of intelligibility. In information retrieval, intelligibility will be key because, in many cases, the information retrieved will be new and unpredictable. The problem is intensified when one considers the likely users of these systems-business and home users who sample a variety of information services in an infrequent and discretionary manner. If these users are unable to understand a synthetic voice, or find it unpleasant, they will simply choose not to use the service. A second set of issues revolves around the manner in which the information is accessed - the database structure and the interface provided to query it. In this paper, we discuss work addressing both sets of issues.

\section{THE INTELLIGIBILITY ISSUE}

The quality of unlimited text-to-speech systems has improved considerably in the last few years. However, significant intelligibility problems still exist. Although Pisoni and Hunnicutt (1980) reported that the recognition of words in meaningful sentences spoken by a good synthesizer is as high as $\mathbf{9 3 . 2 \%}$ correct, performance for semantically anomolous sentences drops to $78.7 \%$ for the same synthesizer. These figures are disturbing, particularly for applications depending on recognition of new, unpredictable information.

The author's mailing address is: Thomas J. Watson Research Center, P.O. Box 218, Yorktown Heights, NY 10598.
One solution to intelligibility problems is to improve the quality of the speech itself, and work on improvements is underway in numerous laboratories (Allen, 1981; Thomas, Klavans, Nartey, Pickover, Reich, \& Rosson, 1984). A complementary approach relies on listeners' ability to learn to better comprehend distorted speech: We know that adaptation occurs for various kinds of distortions, including speech in noise (Tobias \& Irons, 1973), speech produced by deaf speakers (McGarr, 1981; Monsen, 1983), and rule-generated synthesized speech (Schwab, Nusbaum, \& Pisoni, 1983). If we can understand what it is that listeners learn in adapting to speech that has been distorted in some fashion, we can use this understanding to create "training"' environments to maximize adaptation early in a user's interactions with a system.

A first study aimed at understanding adaptation to synthetic speech considered the question of learning generality: To what extent is the learning that takes place specific to the particular sounds a listener is exposed to, and to what extent does it involve the acquisition of some more general language-processing strategy? The answer to this question will have strong implications for the types of adaptation environments likely to be most beneficial.

In the study, listeners were "trained" on a 20-min sentence-transcription task. To manipulate specificity of learning, a listener's training sentences were composed of words representing only a partial set of all available sounds. There were two complementary sets of sounds, and materials were created to incorporate one or the other of the sets. Controls heard the same materials recorded in natural voice. Listeners were then tested on their ability to identify synthesized words never heard before. The test items were all short monosyllabic words, with half composed of sounds from one of the training sets, and half composed of the complementary sounds.

In general, listeners did better both when given synthetic voice training $[\mathrm{F}(1,30)=16.78, \mathrm{p}<.01]$ and when tested on sounds they had heard before $[F(1,30)=10.46$, $\mathrm{p}<.01]$. However, these main effects are qualified by an interaction between training voice, test condition and 
materials set $[F(1,30)=18.10, p<.01]$. Examination of performance on individual sounds revealed that although listeners trained on synthetic speech had their greatest advantage for sounds heard during training, this specific learning effect occurred for a restricted set of sounds: Only some sounds profited from the short-term exposure studied here, with some sounds being uniformly identified well, and with others being identified poorly regardless of training.

These results suggest that one way to facilitate adaptation to a synthetic voice is to identify problematic but "learnable" sounds, and then to give listeners selective exposure to these sounds early in an application's dialog. Research is currently underway to evaluate the effectiveness of such selective exposure in the context of hypothetical instruction paragraphs. We are also exploring the general learning effect observed in an effort to understand how we might exploit it as well.

\section{THE REMOTE-ACCESS INTERFACE}

Much of the appeal in using synthetic voice for remote access lies in the increased availability of the information disbursed, because the common touch-tone phone can serve as the end user's terminal. However, the reliance on such "terminals" poses new problems itself, due to the limitations inherent in the touch-tone keypad. Furthermore, it is by no means clear what data structures and information-retrieval methods will be most effective in this speech-output environment. We have been studying users interacting with a travel information system using speech synthesis in an effort to understand these problems better.

In this work, employees at the T. J. Watson Research Center used a simulated telephone access system containing information about Austin, Texas. The information in the system was organized hierarchically, and we implemented two different command interfaces for cycling through items at a given level, and for moving up and down levels. The verbal mnemonic interface provided commands with meaningful names related to the letters printed on the keys: ' $F$ '" for Forward, ' $B$ '" for Backward, " $M$ " for More Detail, and " $L$ "' for Less Detail. In the spatial mnemonic system, the functions were assigned to keys forming a cross shape, in the hopes of providing a useful spatial metaphor to use in moving right, left, up, and down: The " 6 "' key was used to move Right, the " 4 "' key Left, the " 8 "' key Down, and the " 2 "' key Up. Both systems also allowed users to repeat an utterance, have it played out word by word, and have words spelled.

The users were selected to reflect a wide range of computer sophistication, and included secretaries, administrative workers, technical support staff, and research scientists. They were asked to imagine that they were about to travel to Austin and had called an information number to get some information about the city, such as names, addresses, and phone numbers of eating, shopping, or en- tertainment establishments. Their activity while using the system was logged and time-stamped; we also collected subjective reactions via a postexperiment questionnaire.

The performance data revealed only small differences between the two command interfaces: The verbal mnemonic enjoyed a slight advantage, particularly with respect to users' ability to understand and remember the command descriptions provided by the system at the start of the session [t $(16)=3.06, p<.01]$. This finding suggests that for casual-use applications such as this, in which users must be able to understand and remember a short introductory paragraph describing use of the system, a verbal association for the commands available may be more useful than a spatial layout consistent with the structure of the information base. Of course, such a conclusion must be qualified by the particular implementations developed, because we cannot be certain that some other "spatial" arrangement would not be an even better command interface.

The most striking differences were not between interface conditions, but rather among users, with one able to retrieve the first piece of information in $40 \mathrm{sec}$, and another requiring over $13 \mathrm{~min}$. A number of the users experienced considerable difficulty in navigating the hierarchical structure, with many instances of movement in the wrong direction. Users averaged close to five inappropriate moves per retrieval attempt. This suggests that many users were not applying an appropriate navigational model in searching for information (a suggestion confirmed by informal queries when the experiment was over).

It is important to note that the information needed to convey position in the hierarchy was implicit in the content of the utterances users heard. For example, feedback confirming that one had moved from the top to the middle level was available only by understanding a categorysubcategory relationship. After hearing "Restaurants" and making a Down move, the user might hear "Chinese," and would have to make the inference that a move to a lower level of the hierarchy had been made. This may have been the source of many of the problems, because the most common source of error appeared to arise from users' failure to move laterally to an item once they had reached the appropriate level.

One response to these findings might be to include additional dialog, telling users the effect of each action (e.g., "You have moved to the next item in the Chinese Restaurant list. It is ..."). However, this would add considerable "speech overhead" to the system, probably causing it to appear slow and awkward. More attractive possibilities are to increase the information implicit in the utterance itself, by systematically varying the syntax of the utterances at different levels, or by assigning a different "voice" to each level.

Users' evaluations of the system provided some surprises. They had been asked to evaluate the quality of the speech used (with respect to both intelligibility and pleasantness), and the usefulness and ease of using the 
system. We were not surprised to find relatively low ratings for speech quality: Intelligibility was rated 6.2 out of 10 , and pleasantness 5.1. The surprise came in the effect of these two judgments on the ease-of-use ratings. Users had been asked to judge ease-of-use independently from their reactions to the synthetic speech, in order to better detect differences among the two command interfaces. Furthermore, they were given "anchors" to use in evaluating this novel system-a " 10 " (Very Easy) corresponded to asking an all-knowledgeable friend for the information, and a "1" (Very Difficult) to looking through a randomly ordered book of information. Despite these precautions, however, ease-of-use ratings were related to none of the performance measures: Users experiencing great difficulty with the system were no more likely to give it a poorer rating than those having little or no trouble. Instead, ease of use was solely a function of the perceived intelligibility and pleasantness of the speech.

In general, users felt that a system such as this would be fairly useful (perceived usefulness was rated about 8.5 on a 10-point scale). Unfortunately, it is exactly the novel but critical information (like unusual street names or the names of eating or entertainment establishments) that is very difficult to understand with existing-quality synthesizers. The finding that users' judgments about ease of use were related to speech quality and not to performance undoubtedly reflects the tremendous impact of these intelligibility problems on perceived ability to use the system. If a target utterance cannot be understood, then the number of commands required to retrieve it may seem irrelevant. Such a finding points back to the work described in the first section of the paper, underscoring the importance of working in parallel on intelligibility and user interface issues.

\section{REFERENCES}

Allen, J. (1981). Linguistic-based algorithms offer practical text-tospeech systems. Speech Technology, 1, 12-16.

MCGARR, N. S. (1981). The effect of context on the intelligibility of hearing and deaf children's speech. Language and Speech, 24, 255-264.

MONSEN, R. B. (1983). The oral speech intelligibility of hearing-impaired talkers. Journal of Speech and Hearing Disorders, 48, 286-296.

PISONI, D. B., \& HunNicutT, S. (1980). Perceptual evaluation of MITalk: The MIT unrestricted text-to-speech system. In Proceedings of the International Conference on Acoustics, Speech, and Signal Processing (Report No. CM15594; pp. 572-575). Piscataway, NY: IEEE Service Center.

Schwab, E. C., Nusbaum, H. C., \& Pisoni, D. B. (1983). Some effects of training on the perception of synthetic speech. Manuscript submitted for publication.

Thomas, J., Klavans, J., Nartey, J., Pickover, C., Reich, D., \& Rosson, M. B. (1984). WALRUS: A development system for speech synthesis (IBM Research Rep. No. RC 10626). Yorktown Heights, NY: Watson Research Center.

TOBIAS, J. V., \& IRONS, F. M. (1973). Reception of distorted speech (FAA Rep. No. FAA-AM-73-13). Oklahoma City, OK: FAA Civil Aeromedical Institute. 